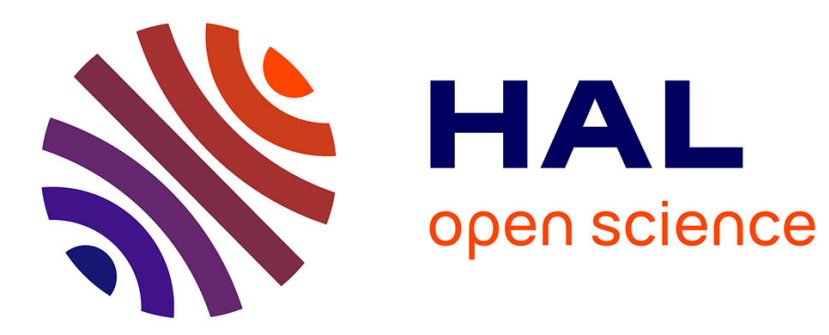

\title{
Role of elongational viscosity of feedstock in extrusion-based additive manufacturing of powder-binder mixtures
}

Kedarnath Rane, Thierry Barriere, Matteo Strano

\section{- To cite this version:}

Kedarnath Rane, Thierry Barriere, Matteo Strano. Role of elongational viscosity of feedstock in extrusion-based additive manufacturing of powder-binder mixtures. International Journal of Advanced Manufacturing Technology, 2020, 107 (11-12), pp.4389 - 4402. hal-03020955

\section{HAL Id: hal-03020955 https://hal.science/hal-03020955}

Submitted on 24 Nov 2020

HAL is a multi-disciplinary open access archive for the deposit and dissemination of scientific research documents, whether they are published or not. The documents may come from teaching and research institutions in France or abroad, or from public or private research centers.
L'archive ouverte pluridisciplinaire HAL, est destinée au dépôt et à la diffusion de documents scientifiques de niveau recherche, publiés ou non, émanant des établissements d'enseignement et de recherche français ou étrangers, des laboratoires publics ou privés. 


\section{Dear Author}

Here are the proofs of your article.

- You can submit your corrections online or by fax.

- For online submission please insert your corrections in the online correction form. Always indicate the line number to which the correction refers.

- Please return your proof together with the permission to publish confirmation.

- For fax submission, please ensure that your corrections are clearly legible. Use a fine black pen and write the correction in the margin, not too close to the edge of the page.

- Remember to note the journal title, article number, and your name when sending your response via e-mail, fax or regular mail.

- Check the metadata sheet to make sure that the header information, especially author names and the corresponding affiliations are correctly shown.

- Check the questions that may have arisen during copy editing and insert your answers/corrections.

- Check that the text is complete and that all figures, tables and their legends are included. Also check the accuracy of special characters, equations, and electronic supplementary material if applicable. If necessary refer to the Edited manuscript.

- The publication of inaccurate data such as dosages and units can have serious consequences. Please take particular care that all such details are correct.

- Please do not make changes that involve only matters of style. We have generally introduced forms that follow the journal's style.

Substantial changes in content, e.g., new results, corrected values, title and authorship are not allowed without the approval of the responsible editor. In such a case, please contact the Editorial Office and return his/her consent together with the proof.

- If we do not receive your corrections within $\mathbf{4 8}$ hours, we will send you a reminder.

\section{Please note}

Your article will be published Online First approximately one week after receipt of your corrected proofs. This is the official first publication citable with the DOI. Further changes are, therefore, not possible.

After online publication, subscribers (personal/institutional) to this journal will have access to the complete article via the DOI using the URL:

http://dx.doi.org/10.1007/s00170-020-05323-9

If you would like to know when your article has been published online, take advantage of our free alert service. For registration and further information, go to: http://www.springerlink.com.

Due to the electronic nature of the procedure, the manuscript and the original figures will only be returned to you on special request. When you return your corrections, please inform us, if you would like to have these documents returned.

The printed version will follow in a forthcoming issue. 


\section{Metadata of the article that will be visualized in OnlineFirst}

\begin{tabular}{|c|c|c|c|}
\hline 1 & Article Title & \multicolumn{2}{|c|}{$\begin{array}{l}\text { Role of elongational viscosity of feed stock in ex trusion-based additive } \\
\text { manufacturing of powder-binder mix tures }\end{array}$} \\
\hline 2 & \multicolumn{3}{|l|}{ Article Sub- Title } \\
\hline 3 & $\begin{array}{l}\text { Article Copyright - } \\
\text { Year }\end{array}$ & \multicolumn{2}{|c|}{$\begin{array}{l}\text { Springer-Verlag London Ltd., part of Springer Nature } 2020 \\
\text { (This will be the copyright line in the final PDF) }\end{array}$} \\
\hline 4 & Journal Name & \multicolumn{2}{|c|}{ The International Journal of Advanced Manufacturing Technology } \\
\hline 5 & \multirow{8}{*}{$\begin{array}{l}\text { Corresponding } \\
\text { Author }\end{array}$} & Family Name & Strano \\
\hline 6 & & Particle & \\
\hline 7 & & Given Name & Matteo \\
\hline 8 & & Suffix & \\
\hline 9 & & Organization & Politecnico di Milano \\
\hline 10 & & Division & Dipartimento di Meccanica \\
\hline 11 & & Address & Via La Masa 1, Milan 20159, Italy \\
\hline 12 & & e-mail & matteo.strano@polimi.it \\
\hline 13 & \multirow{8}{*}{ Author } & Family Name & Rane \\
\hline 14 & & Particle & \\
\hline 15 & & Given Name & Kedarnath \\
\hline 16 & & Suffix & \\
\hline 17 & & Organization & Politecnico di Milano \\
\hline 18 & & Division & Dipartimento di Meccanica \\
\hline 19 & & Address & Via La Masa 1, Milan 20159, Italy \\
\hline 20 & & e-mail & \\
\hline 21 & \multirow{8}{*}{ Author } & Family Name & Barriere \\
\hline 22 & & Particle & \\
\hline 23 & & Given Name & Thierry \\
\hline 24 & & Suffix & \\
\hline 25 & & Organization & Univ. Bourgogne Franche-Comté, FEMTO-ST Institute \\
\hline 26 & & Division & $\begin{array}{l}\text { CNRS/UFC/ENSMM/UTBM, Department of Applied } \\
\text { Mechanics }\end{array}$ \\
\hline 27 & & Address & Besançon 25000, France \\
\hline 28 & & e-mail & \\
\hline 29 & \multirow{2}{*}{ Schedule } & Received & 17 December 2019 \\
\hline 30 & & Revised & \\
\hline
\end{tabular}



manufacturing (EAM) technique and has received significant interest. EAM feedstocks are generally characterized by their shear viscosity. A quantitative comparison with the shear flow data, through an estimation of the Trouton ratio, indicates that the extensional viscosities are three orders of magnitude greater than their shear flow viscosity at a comparable shear rate obtained in three different high loaded polymers retained for this study. This experimental study addresses the unsolved issue of the role of elongational viscosity in the modelling of EAM of highly viscous melts. The study was conducted using three feedstocks with a water-soluble binder and high powder loading. The different powder materials used for this study are stainless steel, alumina and zirconia. Initially, the rheological properties of the feedstocks were assessed using capillary rheometers. A pressure drop model based on the shear and elongational components of the viscosity was proposed to predict the extrusion pressure during capillary tests. The model was adapted to develop a specific EAM machine, namely, an EFeSTO, equipped with a pellet extrusion unit. Experimental EAM tests were conducted, and the pressure drops were analytically predicted and experimentally measured. A total of 31 different combinations of extrusion velocities, nozzle diameters, 3D printed shapes and materials were tested through a total 184 experimental runs. The model predicts well the experimental pressures for the steel feedstock, whereas it underestimates the pressure for the two ceramic feedstocks owing to their different thermal properties. The results of this study clearly demonstrate that the pressure, and therefore the material flow during the EAM processes of viscous materials, cannot be modelled well without considering the elongational viscosity.

33 Keywords separated 3D printing - Highly viscous melt - Extrusion pressure - Elongational by ' - ' viscosity

34 Foot note Springer Nature remains neutral with regard to jurisdictional claims in information published maps and institutional affiliations. 
4

\title{
Role of elongational viscosity of feedstock in extrusion-based additive manufacturing of powder-binder mixtures
}

\author{
Kedarnath Rane ${ }^{1} \cdot$ Thierry Barriere $^{2} \cdot$ Matteo Strano $^{1}$ \\ Received: 17 December 2019 / Accepted: 13 April 2020 \\ (C) Springer-Verlag London Ltd., part of Springer Nature 2020
}

\begin{abstract}
The 3D printing of metals and ceramics by the extrusion of a powder/thermoplastic binder feedstock is an extrusion-based additive manufacturing (EAM) technique and has received significant interest. EAM feedstocks are generally characterized by their shear viscosity. A quantitative comparison with the shear flow data, through an estimation of the Trouton ratio, indicates that the extensional viscosities are three orders of magnitude greater than their shear flow viscosity at a comparable shear rate obtained in three different high loaded polymers retained for this study. This experimental study addresses the unsolved issue of the role of elongational viscosity in the modelling of EAM of highly viscous melts. The study was conducted using three feedstocks with a water-soluble binder and high powder loading. The different powder materials used for this study are stainless steel, alumina and zirconia. Initially, the rheological properties of the feedstocks were assessed using capillary rheometers. A pressure drop model based on the shear and elongational components of the viscosity was proposed to predict the extrusion pressure during capillary tests. The model was adapted to develop a specific EAM machine, namely, an EFeSTO, equipped with a pellet extrusion unit. Experimental EAM tests were conducted, and the pressure drops were analytically predicted and experimentally measured. A total of 31 different combinations of extrusion velocities, nozzle diameters, 3D printed shapes and materials were tested through a total 184 experimental runs. The model predicts well the experimental pressures for the steel feedstock, whereas it underestimates the pressure for the two ceramic feedstocks owing to their different thermal properties. The results of this study clearly demonstrate that the pressure, and therefore the material flow during the EAM processes of viscous materials, cannot be modelled well without considering the elongational viscosity.
\end{abstract}

Keywords $3 \mathrm{D}$ printing $\cdot$ Highly viscous melt $\cdot$ Extrusion pressure $\cdot$ Elongational viscosity

\section{Introduction}

Powder injection moulding (PIM) is a convenient widespread manufacturing process for producing complex components in large batches [1]. It employs a feedstock usually composed of a thermoplastic polymeric binder, filled with metals or a ceramic powder. This type of feedstock can also be used for extrusion-based additive manufacturing (EAM) technologies for metallic and ceramic components [2]. There are two types

Matteo Strano

matteo.strano@polimi.it

1 Dipartimento di Meccanica, Politecnico di Milano, Via La Masa 1, 20159 Milan, Italy

$2 \mathrm{CNRS/UFC/ENSMM/UTBM,} \mathrm{Department} \mathrm{of} \mathrm{Applied} \mathrm{Mechanics,}$ Univ. Bourgogne Franche-Comté, FEMTO-ST Institute, 25000 Besançon, France of EAM technologies. One is called direct ink writing (DIW), which has also been called robocasting, based on the direct use of a powder-binder/matrix slurry feedstock (without increasing the temperature for melting) [3]. The other is called fused filament deposition (FFD). It is a $3 \mathrm{D}$ printing process or additive manufacturing technique that applies a continuous filament. A filament-type thermoplastic polymer is melted before it extrudes from the nozzle and is deposited on the growing specimen. The headed printer extruder heat usually moves in two dimensions to deposit one horizontal layer at a time. The specimen or printer extruder head is then moved vertically by a small amount to begin a new layer. To realize a 3D component with a functional material, Nadernezhad et al. [4] investigated the extrusion of PLA/CNT nanocomposites dedicated to additive manufacturing using this FFD process. In our case, the FFD process has been modified for application with pellets instead of a filament using the EAM of powderbinder mixtures. 
Some EAM machines for the processing of such feedstocks are commercially available (including Markforged Metal X and Desktop Metal Studio); however, no commercial machines are yet available for extrusion starting from the pellets of feedstock, instead of filaments or rods. EAM machines based on the extrusion of pellets allow for material diversity and are cost-effective.

The EFeSTO machine has been previously developed and was employed in this study. It combines a servo-controlled small pellet extruder unit with a robotic deposition table based on parallel kinematics [5]. One advantage of EFeSTO for the present study is that the torque (and therefore the pressure) applied by the pellet extrusion unit can be monitored during the processing.

Melt viscosity [6] is one of the important characteristics of a feedstock and is used to predict the rheological behaviour during highly viscous melt extrusion and in the correct design of a 3D printing process through the selection of appropriate extrusion parameters.

In previous studies associated with powder injection moulding, the rheology of highly loaded feedstocks has generally been assessed through a capillary rheometer, used to characterize the shear viscosity behaviour [7]. A literature review dedicated to the laws of highly concentrated feedstock alloys is available in [8], where a shear viscosity model was proposed for superalloy powders. A capillary rheometer is generally preferred over other rheometers to reduce the estimation errors from a wall slip [9]. The shear viscosity is universally and correctly considered the most important parameter for highly viscous PIM feedstocks. However, during the EAM processes, the shear rates are comparably smaller, and the extrusion nozzles are shorter; therefore, compared with PIM, EAM processes induce a comparably lower amount of shear deformation, whereas the extruded filaments inevitably elongate. In a review on the EAM processes [10], the extensional viscosity was not mentioned. In a more recent review [11], the author recognized that elongational viscosity is generally accepted as an important parameter for determining the pressure drops in additive manufacturing through a material extrusion. However, despite this common belief, the characterization of the elongational viscosity in scientific papers dealing with the EAM processes has generally been neglected. In [12], the authors list all of the relevant feedstock properties for the EAM of metals, and place a large emphasis on the shear viscosity, while neglecting to mention the elongational component. The shear viscosity is still frequently considered a unique or important property of highly viscous EAM feedstocks, such as in [13], where the authors studied the EAM of zirconia, or in [14], where the authors studied the effects of the powder size on the properties of highly filled polymers for fused filament deposition (FFD). In [15], the authors characterized the viscosity of highly viscous polymers for FFD and recognized the importance of the material at extremely small or "zero" shear rates; nevertheless, they modelled and represented the shear viscosity only, and not the elongational viscosity.

One of the reasons why the elongational or entrance viscosity of viscous non-Newtonian fluids during the EAM processes has been neglected by the scientific literature is the inherent difficulty of knowing the instantaneous extrusion pressure during such processes or, even worse, the instantaneous shear stress. In typical FFD machines, the instantaneous extrusion pressure is unknown. As an exception, in [16] the authors conducted a very interesting study using in-line rheological pressure measurements in FFD. However, they did not characterize or isolate the extensional viscosity.

The purpose of the present study is to demonstrate that, for EAM with specific viscous melts, which take place at a low shear rate and within relatively short extrusion nozzles, the characterization of the feedstock based on the elongational viscosity is more important than the shear viscosity when predicting the flow.

The remainder of this paper is organized as follows: In the next section, the relevant rheological models are presented, highlighting the differences between the shear and elongational viscosity. The experimental materials, methods and equipment are then described. In the third section, the rheological model is validated based on capillary rheometer data. Finally, the results of extrusion and $3 \mathrm{D}$ printing tests using EFeSTO equipment are presented and discussed.

\section{Rheological models}

The rheology of powder-binder feedstocks has been extensively studied, and many models have been proposed to describe the melt viscosity during the extrusion and injection moulding processes. The well-known constitutive equation for the shear viscosity of Newtonian fluids is as follows:

$\eta_{\mathrm{s}}=\frac{\tau}{\dot{\gamma}}$

where $\tau$ is the shear stress and $\dot{\gamma}$ is the applied shear rate. In addition, $\eta_{\mathrm{s}}$ is the shear viscosity or the resistance of the fluid to shearing. The shear viscosity is a constant for Newtonian fluids, whereas the powder-binder feedstocks usually show a non-Newtonian characteristic [17]. In PIM applications, a shear-thinning (or pseudoplastic) effect is observed, where the shear viscosity decreases upon an increase in the shear rate [18]. The simplest way to describe a pseudoplastic effect is the power law model, which demonstrates a non-linear relation between the shear stress and shear rate as follows:

$\tau=K \gamma^{\cdot n}$ 
where $K$ and $n$ are material-specific parameters, namely, the consistency and shear rate sensitivity, respectively. The shear rate sensitivity $n$ is the power law index, which is $n<1$ for pseudoplastic fluids; shear thinning then becomes more evident with a decrease in $n$. In a previous study [6], it was demonstrated that larger $K$ values favour a better stability of the extrusion of the metal-binder feedstocks, in terms of both the pressure signal and filament quality.

Experimental measurements of the shear viscosity can be conducted using a variety of instruments, given the wide range of viscosities that feedstock materials can present [19]. The most common are capillary rheometers, which can be used from 2 to $3000 s^{-1}$ [20]. For a capillary rheometer, pressure is applied using a piston, and the apparent shear rate $\left(\dot{\gamma}_{\mathrm{a}}\right)$ and shear stress at the wall $\left(\tau_{\mathrm{w}}\right)$ are determined from the extruded flow rate for non-Newtonian fluids:

$\tau_{\mathrm{w}}=\frac{\Delta P_{\text {cap }}}{L / 2 R}$

$\dot{\gamma}_{\mathrm{a}}=\frac{4 Q}{\pi R^{3}}$

where $\Delta P_{\text {cap }}$ is the pressure drop at the capillary, $L$ is the capillary length, $R$ is the radius, $Q$ is the volumetric flow rate and $\dot{\gamma}_{\mathrm{a}}$ is the apparent shear rate, i.e. the true shear rate of a Newtonian fluid. For shear-thinning fluids, Rabinowitsch correction for determining a more realistic value of the true shear rate $\dot{\gamma}_{\mathrm{w}}$ must be employed [21]:

$\dot{\gamma}_{\mathrm{w}}=\frac{(3 n+1) 4 Q}{4 n \pi R^{3}}$

Nozzles used in EAM machines for highly viscous polymers [2] are generally extremely short, with length over diameter (L/D) ratios of well below 10 . For short capillaries $(L / D<$ 25), an additional pressure drop $\Delta P_{\mathrm{e}}$ at the entrance must be accounted for owing to the sharp decrease in diameter from the barrel where the material is compressed before entering the capillary. Bagley's correction is often used for this purpose [17]:

$n_{\mathrm{B}}=\frac{\Delta P_{\mathrm{e}}}{2 \tau_{\mathrm{w}}}$

Bagley's corrected shear stress at the wall can be calculated as follows:

$\tau_{\mathrm{w}}=\frac{\left(\Delta P_{\text {cap }}+\Delta P_{\mathrm{e}}\right)}{2\left({ }^{L} /{ }_{R}+n_{\mathrm{B}}\right)}$

Bagley's correction depends on both the geometry of the capillary and the material characteristics. The role of the entrance pressure drop in the short capillaries has been considered by many authors to be related to the so-called elongational or extensional viscosity [22]. Indeed, for a deeper understanding of the rheological of the feedstock during the extrusion process of EAM, the contributions of the shear viscosity and the elongational viscosity need to be explicitly quantified. Numerous models have been proposed to describe the elongational viscosity of polymer melts, e.g. using a flow through a tube with an abrupt contraction as a measure [23]. For elongational rheometry experiments of non-Newtonian fluids, the elastic and viscous contributions can be separated [24]. When characterizing highly viscous materials, the roles of the capillarity and gravity are generally neglected.

One of the most accredited models for estimating the entrance pressure drop $\left(\Delta P_{\mathrm{e}}\right)$ was developed by Cogswell [25], who assumed that the pressure drop can be modelled by defining the shear viscosity $\left(\eta_{\mathrm{s}}\right)$ and elongational viscosity $\left(\eta_{\mathrm{E}}\right)$ dependent terms [26]. This model is only accurate at low deformation rates (as in EAM applications). As an alternative to Cogswell's model, Binding and Gibson's model [27] can also be used to accurately describe the pseudoplastic effect of PIM feedstock over a wide range of shear rates when considering the contributions of the shear and elongational viscosity. A simple rheological model, comparable with Binding and Gibson's model, is proposed herein to analyse the results of twin-bore capillary rheometers.

\subsection{Rheology of feedstock: shear and elongational viscosities}

Polymer processing through the mixing and printing of a high loaded polymer usually involves medium and large strain rates in shear and extensional flows, and the viscosity of the feedstock depends on the binder composition and properties of the powders, mixing parameters and conditions. In the case of a high loaded polymer, Arabo [28] concluded that an extensional (or elongational) flow is important and has therefore attracted significant interest in the powder forming processes.

In a twin-bore rheometer, there are two nozzles: The nozzle on the left is a long capillary (L/D>> 10) and the nozzle on the right has a negligible length, i.e. virtually a "zero shear" deformation. A simple model has been developed based on the data obtained from a twin-bore capillary rheometer. This model was then validated, as shown later in this paper, on a different twin-bore rheometer with a different L/D ratio. The model assumes that the total pressure at a long (left) capillary is considered the sum of two components as follows:

$P_{\text {tot }}=\Delta P_{\text {left }}=\Delta P_{\text {ent }}+\Delta P_{\text {cap }}$,

where $\Delta P_{\text {ent }}$ is the entrance pressure variation owing to the abrupt change in section between the barrel and capillary. This $\Delta P_{\text {ent }}$ value can therefore be directly measured from the right bore, which is associated with the calculation of 
the elongational viscosity $\left(\eta_{\mathrm{E}}\right)$ and elongational strain rate $\varepsilon$. The second term $\Delta P_{\text {cap }}$ is associated with the shear deformation and shear viscosity, which can be calculated at the long (left) bore after subtracting $\Delta P_{\text {ent }}$. True corrections have been applied to the capillary rheometer data to more accurately describe the non-Newtonian behaviour of the feedstock, namely, the above-mentioned Bagley's and Rabinowitsch corrections. Moreover, owing to the high viscosity of the powder-binder feedstock, the assumption of no wall slipping, typical of capillary rheology, is removed. Therefore, the apparent shear rate was corrected as follows:

$\dot{\gamma}=\dot{\gamma}_{a}-\dot{\gamma}_{0}$

where $\dot{\gamma}_{0}$ is an experimental constant determined by the least squares minimisation; this expresses the shear rate reduction owing to a wall slip. The shear viscosity $\left(\eta_{\mathrm{s}}\right)$ is modelled using a power law equation as a function of the corrected shear strain:

$\eta_{\mathrm{s}}=K \dot{\gamma}^{n-1}$

The right capillary provides a negligible shear resistance, and therefore its pressure reading, the major cause of which is the entrance pressure, can be entirely associated with the elongational viscosity $\left(\eta_{\mathrm{E}}\right)$ :

$\eta_{\mathrm{E}}=\frac{\sigma_{E}}{\dot{\varepsilon}}$,

where $\sigma_{\mathrm{E}}$ is the elongational stress at the orifice and $\varepsilon$ is the elongational strain rate. The elongational viscosity can also be modelled using a power law equation as a function of the apparent shear rate:

$\eta_{\mathrm{E}}=l \gamma_{a}^{\cdot y-1}$

where $l$ and $y$ are the consistency and sensitivity parameters associated with the elongational viscosity. The elongational strain rate is independent of the capillary length, although the capillary diameter does have an influence. For a given apparent shear rate $\dot{\gamma}_{\mathrm{a}}$, the elongational strain rate can be estimated based on the following:

$\dot{\varepsilon}=\frac{\dot{\gamma}_{a}}{4}$

Under Cogswell's model assumptions, the elongational stress can be calculated as a function of the entrance pressure drop as follows:

$\sigma_{\mathrm{E}}=\frac{3}{8}(n+1) \Delta P_{\mathrm{ent}}$.
After substituting the terms $\eta_{\mathrm{s}}, \eta_{\mathrm{E}}, \gamma, \varepsilon$ and $\sigma_{\mathrm{E}}$ in Eq. (8), the total pressure drop in the left capillary can be finally expressed in the following way:

$$
\begin{aligned}
P_{\text {tot }} & =\Delta P_{\text {cap }}+\Delta P_{\text {ent }}=\eta_{s} \cdot \frac{4 L_{l}}{D}+\eta_{\mathrm{E}} \dot{\gamma}_{\mathrm{a}} \frac{2}{3(n+1)} \\
& =K \gamma^{n} \frac{4 L_{l}}{D}+1 \dot{\gamma}_{\mathrm{a}}^{y} \frac{2}{3(n+1)}
\end{aligned}
$$

\section{Materials, equipment and methods}

\subsection{Feedstock characterization}

Three different feedstocks were used for this study. A feedstock with a solid loading of stainless steel (SS 316L) powder was prepared by mixing a water-soluble Embemould K83 binder (eMBe, Gmbh) and gas-atomised (SS 316L) powder (Sandvik Osprey) in a Brabender Plasti-Corder mixer. Parenti et al. [29] used the same binder for thermoplastic processing, applying a combination based on polymers with water-soluble components. The binder is specifically devoted to aqueous debinding for the PIM process. After a DSC analysis, they concluded that it is multi-constituent with three different ingredients and that the highest associated melt temperature is approximately $118^{\circ} \mathrm{C}$. The density of the water-soluble material $\mathrm{K} 83$ is $1.05 \mathrm{~g} / \mathrm{cm}^{3}$. Mixing of the $\mathrm{K} 83$ binder and powder was performed at $145{ }^{\circ} \mathrm{C}$ for $30 \mathrm{~min}$ to produce a homogeneous feedstock without introducing air bubbles.

This feedstock mixture was further processed through a twin-screw extruder at $145^{\circ} \mathrm{C}$ to obtain a highly homogeneous and pelletised feedstock for the subsequent operations. Two commercial (INMATEC, Gmbh) ceramic feedstocks, having a solid loading of alumina-based ceramic powder (INMAFEED K1008) and zirconia-based ceramic powder (INMAFEED K1009), were procured. The chemical composition of the stainless steel, alumina and zirconia powders is shown in Table 1.

In Table 2, the relevant physical and thermal properties of the investigated feedstocks are shown. Physical and thermal properties of the feedstock play an important role in the stability and phase change during extrusion and 3D printing.

All powders used in the present study are fine powders with $d_{50}$ of less than $10 \mu \mathrm{m}$, allowing components with a fine microstructure and smooth surface to be produced through the EAM process.

The volumetric powder loading $\varphi$ of the two types of commercial ceramic feedstock was clearly selected by the producer. The powder loading of the steel feedstock with the best value for extrudability was selected according to a previous study [6]. 
Int J Adv Manuf Technol

t1.1 Table 1 Chemical composition (by wt $\%$ ) of powders used in the present study

\begin{tabular}{llllllllll} 
t1.2 & Element & $\mathrm{Cr}$ & $\mathrm{Ni}$ & $\mathrm{Mo}$ & $\mathrm{Mn}$ & $\mathrm{Si}$ & $\mathrm{C}$ & $\mathrm{P}$ & $\mathrm{S}$ \\
\cline { 2 - 8 } $\mathrm{t} 1.3$ & SS 316L steel & 17.90 & 11.70 & 2.30 & 1.41 & 0.72 & 0.02 & 0.02 & 0.006 \\
$\mathrm{t} 1.4$ & Compound & $\mathrm{Na}_{2} \mathrm{O}$ & $\mathrm{Fe}_{2} \mathrm{O}_{3}$ & $\mathrm{SiO}_{2}$ & $\mathrm{Al}_{2} \mathrm{O}_{3}$ & $\mathrm{ZrO}_{2}$ & $\mathrm{Y}_{2} \mathrm{O}_{3}$ & $\mathrm{MgO}$ & $\mathrm{CaO}$ \\
$\mathrm{t} 1.5$ & Alumina & 0.1 & 0.03 & 1.8 & 96 & - & - & 0.9 & 1.3 \\
$\mathrm{t} 1.6$ & Zirconia & 0.04 & $0.01 \%$ & 0.02 & 0.25 & 94.5 & 5.15 & - & - \\
\cline { 3 - 8 }
\end{tabular}

The thermal conductivity $(k)$ of the feedstock is nonproportional to the solid content (i.e. weight of the powder in the feedstock) because the heat flow is limited by the binder system, with a continuous matrix forming a layer between particles. The thermal conductivity $k$ of each feedstock was calculated using an equation provided by Lobo and Cohen [30]:

$\frac{1}{k}=\frac{1-\varphi}{k_{\mathrm{b}}}+\frac{\varphi}{k_{\mathrm{f}}}$,

where $\varphi$ is the volumetric powder loading and $k_{\mathrm{b}}$ and $k_{\mathrm{p}}$ are the nominal thermal conductivities of the binder and powder, respectively.

The heat capacity $C_{\mathrm{p}}$ of the feedstock is calculated up to the suggested operational temperature $\left(145^{\circ} \mathrm{C}\right.$ for alumina, $175^{\circ} \mathrm{C}$ for zirconia and $130{ }^{\circ} \mathrm{C}$ for stainless steel), through an analysis of the DSC curve [31]. The three powders differ considerably in terms of the heat capacity, the estimate of which is also provided in Table 2. The values were measured based on differential scanning calorimetry (DSC) tests, conducted under the ASTM D3418-15 standard, using a differential scanning calorimeter (DSC2010, TA Instruments). The pan was aluminium, and the test was performed in a nitrogen atmosphere, with a gas flow rate of $40 \mathrm{ml} / \mathrm{min}$. The applied heating rate corresponded to $5^{\circ} \mathrm{C} / \mathrm{min}$. The DSC curves of the feedstocks are plotted in Fig. 1, which show a comparison between the curves of the metal and the ceramic feedstock. Both ceramic feedstocks present a peak at the same temperature of $60.8{ }^{\circ} \mathrm{C}$ corresponding to the melting point of the industrial binder. However, the stainless steel feedstock presents a single fusion peak at $62.9^{\circ} \mathrm{C}$ with a latent heat of fusion of $26.1 \mathrm{~J} / \mathrm{g}$. The solidification temperature is observed to be $38.0^{\circ} \mathrm{C}$. The DSC curve also shows indistinct peaks at $64.4^{\circ} \mathrm{C}, 96.9^{\circ} \mathrm{C}, 110.5^{\circ} \mathrm{C}$ and $158.3^{\circ} \mathrm{C}$, corresponding to the melting and solidification of different components (PEG, PMMA, surfactants and additives, respectively) in an Embemould K83 binder.

Once the properties $\rho, C_{\mathrm{p}}$ and $k$ are known, the diffusivity $\alpha$ can be calculated as a derived variable. Table 2 shows that the thermal diffusivity $\alpha$ of zirconia is the largest (owing to its low heat capacity), followed by alumina, whereas the diffusivity of the steel feedstock is significantly smaller (owing to its higher density).

\subsection{Capillary rheometers}

The rheological properties of all feedstocks were determined using two different twin-bore capillary rheometers (Fig. 2a), labelled as rheometers A and B (Malvern Panalytical). The selected test temperatures were $145{ }^{\circ} \mathrm{C}$ for alumina, $175{ }^{\circ} \mathrm{C}$ for zirconia and $130{ }^{\circ} \mathrm{C}$ for stainless steel, over a wide range (50 to $1000 \mathrm{~s}^{-1}$ ) of shear rates. A sample of each material was positioned in a cylindrical barrel with moving pistons. Defining the piston speed, the material is forced into a long capillary of known diameter $D_{1}$ and length $L_{1}$ at the bottom left of the barrel and into an extremely short capillary with $D_{\mathrm{r}}$ and $L_{\mathrm{r}}$ on the right. The values of $D_{\mathrm{r}}$ and $L_{\mathrm{r}}$ for both rheometers are given in Fig. 2c and used for the calculations described in Sect. 4.1 for validation. Pressure transducers are placed immediately above the capillaries; the output of this test is therefore the pressure from each bore. This setup allows the determination of the shear viscosity from the left capillary and the elongational viscosity from the right capillary, according to the model presented above.
386 387 388 389 390 391 392 393 394 395 396 397 398 399 400 401 402 403 404
Table 2 Physical and thermal properties of feedstock used in the present study

\begin{tabular}{lllllll}
\hline Feedstock & $d_{50}(\mu \mathrm{m})$ & $\varphi($ vol $\%)$ & $\rho\left(\mathrm{kg} / \mathrm{m}^{3}\right)$ & $\mathrm{k}(\mathrm{W} / \mathrm{m} \mathrm{K})$ & $C_{\mathrm{p}}(\mathrm{J} / \mathrm{kg} \mathrm{K})$ & $\alpha(\mathrm{vol} \%)$ \\
\hline $\mathrm{Al}_{2} \mathrm{O}_{3}$-binder & 1.9 & 60 & 2400 & 0.63 & 1528 & 0.17 \\
$\mathrm{ZrO}_{2}$-binder & 0.6 & 47 & 2550 & 0.43 & 794 & 0.21 \\
$\mathrm{SS} 316 \mathrm{~L}$-binder & 8.8 & 62 & 5320 & 0.66 & 1668 & 0.07 \\
\hline
\end{tabular}

$d_{50}$ is the mean diameter of the powder; $\varphi$ is the powder loading (vol\%) in the feedstock; and $\rho, k$ and $C_{\mathrm{p}}$ are the density, thermal conductivity and heat capacity of the feedstock, respectively 
Fig. 1 DSC curves of alumina, zirconia and stainless steel feedstocks

\subsection{Description of specific EFeSTO equipment}

The EFeSTO machine, shown in Fig. 2b, has been used both for extrusion and $3 \mathrm{D}$ printing tests. The work table is free to

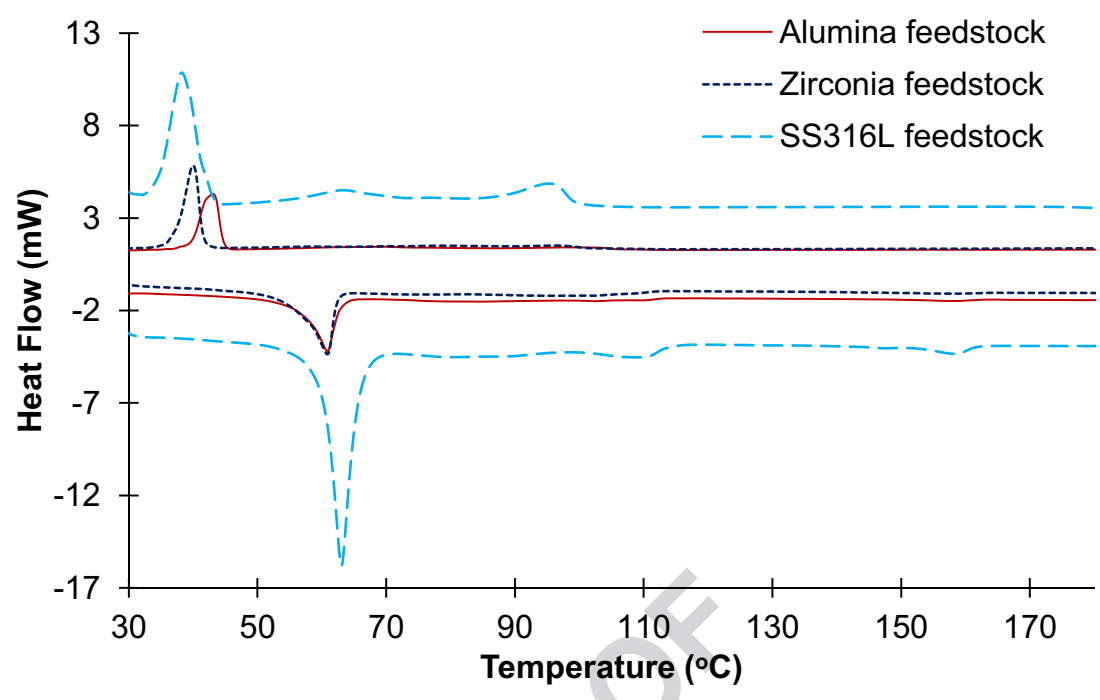

move in the $X$-, $Y$ - and $Z$-directions and is governed by a 3 -axis parallel kinematics linear delta system. The printing head is stationary and composed of a feeder where the pellets of the feedstock are placed, as well as a screw plasticiser and an
408 409 410 411

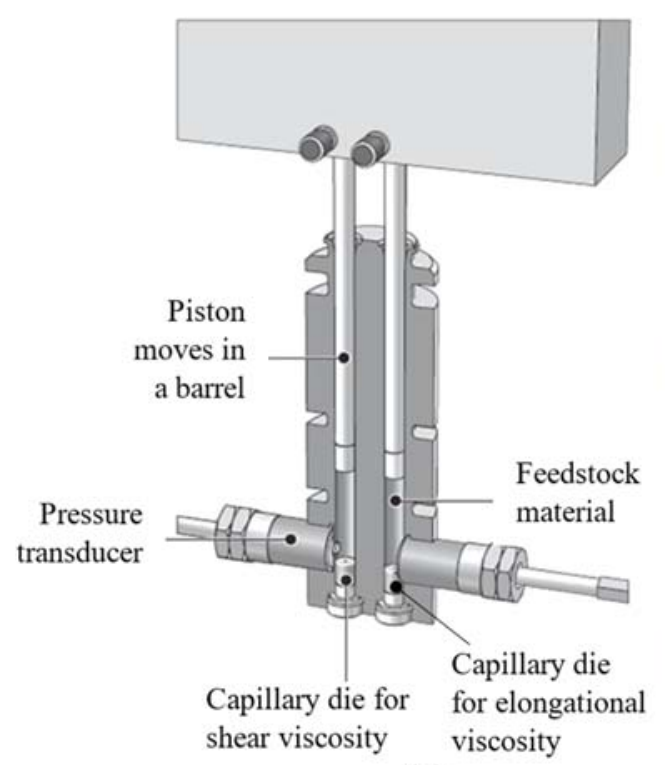

(a)

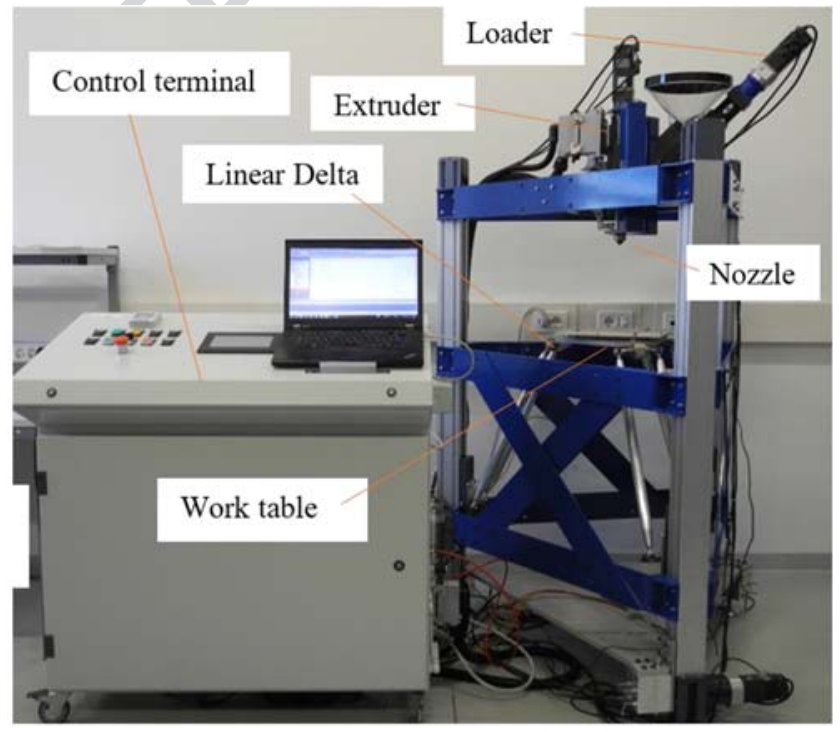

(b)

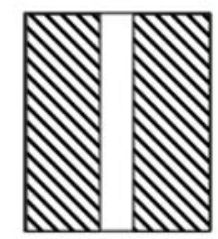

Capillary left die:

$L=32 \mathrm{~mm}$ and

$D_{1}=2 \mathrm{~mm}$

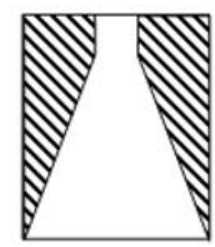

Capillary right die: $L_{r}=0.5 \mathrm{~mm}$ and $D_{r}=2 \mathrm{~mm}$

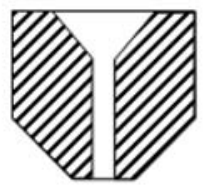

Extrusion nozzle: $L_{n}=4 \mathrm{~mm}$ and $D_{n}=0.8 \mathrm{~mm}$

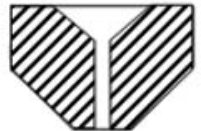

Extrusion nozzle:

$L_{n}=2 \mathrm{~mm}$ and

$D_{n}=0.4 \mathrm{~mm}$

(c)

Fig. 2 Main components of a capillary rheometer and b EFeSTO machine and $\mathbf{c}$ schematic of die and nozzles 
injector piston. In the extruder system, the feedstock is inserted into the feeder and falls into a first loader chamber, which plasticises the material; it is then injected into a second extruder chamber, where a $\mathrm{CNC}$ piston directly pressurizes the melt material through the nozzle. For this study, two different nozzles were employed, with a nozzle diameter $\left(D_{\mathrm{n}}\right)$ of 0.4 and $0.8 \mathrm{~mm}$, respectively.

Three electric resistors (in the plasticisation chamber, in the extrusion chamber and at the nozzle) provide heat to the material, and four thermocouples provide a temperature control. Thermal insulation between the high-temperature plasticisation unit and the actuator unit is achieved using a water-cooling circuit. The stroke of the extrusion piston is synchronized with the g-code of the deposition table and therefore stops during rapid movements of the table, e.g. between consecutive layers of the 3D printed part.

For each experimental run on EFeSTO, the electric current absorbed by the piston drive was recorded and transformed into torque $M$ versus the time signal. Data from the extruder motor was collected using Melsoft MR Configurator software. The data were stored in a local memory support, and owing to the length of the operations, a continuous pressure reading was considered infeasible. Therefore, data were collected during intervals of $50 \mathrm{~s}$ each at different times throughout the tests. The sampling frequency was $20 \mathrm{~Hz}$. During the extrusion and $3 \mathrm{D}$ printing tests, the torque measurements were conducted more frequently at the beginning and end of the tests, with 2 min between consecutive readings. In the central part of each test, the torque was measured with a longer time between readings: $10 \mathrm{~min}$ for extrusion and $5 \mathrm{~min}$ for $3 \mathrm{D}$ printing. The torque $M$ versus time signals were then converted into pressure $P_{\text {tot }}$ signals.

Among the individual samples of the pressure readings, the average total pressure $P_{\text {tot }}$ was calculated along with the standard deviation $S D_{\mathrm{P}}$ and coefficient of variation $C O V_{\mathrm{P}}=P_{\text {tot }} /$ $S D_{\mathrm{P}}$. As an example, in Fig. 3, $P_{\text {tot }}$ is plotted versus time during a sequence of extrusion and printing tests. The typical long-run trend of the pressure signal undergoes an initial increase in pressure and stabilization and a marginal increase at the end of the piston stroke. This is coherent with the flow of pseudoplastic fluids: The initial increase corresponds to an activation of the flow, and stabilization occurs because of the steady-state extrusion regime. The pressure increase at the end of the stroke likely occurs because the piston attempts to extrude the material, which forms a dead zone at the corners of the extrusion chamber.

\subsection{Experimental plan implemented using EFeSTO}

Two main types of tests were conducted: free continuous extrusion tests and 3D printing tests. During each test, as described before, the average extrusion pressure at piston $P_{\text {tot }}$, as well as its standard deviation $S D_{\mathrm{P}}$ and coefficient of variation $C O V_{\mathrm{P}}$, were recorded at regular intervals. During continuous extrusion, the extrusion piston moves at a constant speed, whereas during real 3D printing, it experiences multiple starts and stops, which might influence the measured values of $S D_{\mathrm{p}}$. For each feedstock, three different shapes were 3D printed (shown in Table 3): cylinders with a base diameter of $10 \mathrm{~mm}$ and a height of $10 \mathrm{~mm}$ and bars with a rectangular cross section with a $6 \mathrm{~mm}$ height, $60 \mathrm{~mm}$ length and $10 \mathrm{~mm}$ width. The rectangular bars were printed in both a horizontal and vertical configuration, placed on a face with dimensions of $60 \mathrm{~mm} \times 10 \mathrm{~mm}$.

The parameter settings used for extrusion and 3D printing tests, and designed to produce different apparent shear rates, are given in Table 3, namely, two nozzle diameters $D_{\mathrm{n}}$, three extrusion velocities $V e$, three materials and four types of test. The layer height $h$ does not have an influence on the pressure readings and therefore is not listed in Table 3; however, it was varied around a centre value of half the nozzle diameter. A full factorial experimental plan would have required 72 different experimental conditions, plus replicates. Table 3 lists only 31 out of 72 possible experimental conditions, which were used to keep the experimental cost within a reasonable limit. The 31 tested conditions were replicated a minimum of 2 and a maximum of 5 times, for a total of 184 different tests. Multiple
Fig. 3 Sequence of pressure readings for extrusion pressure $\left(P_{\text {tot }}\right)$ of stainless steel feedstock: average measured pressure $P_{\text {tot }}=$ 3.86 MPa with coefficient of variation $C O V_{\mathrm{P}}=8.1 \%$

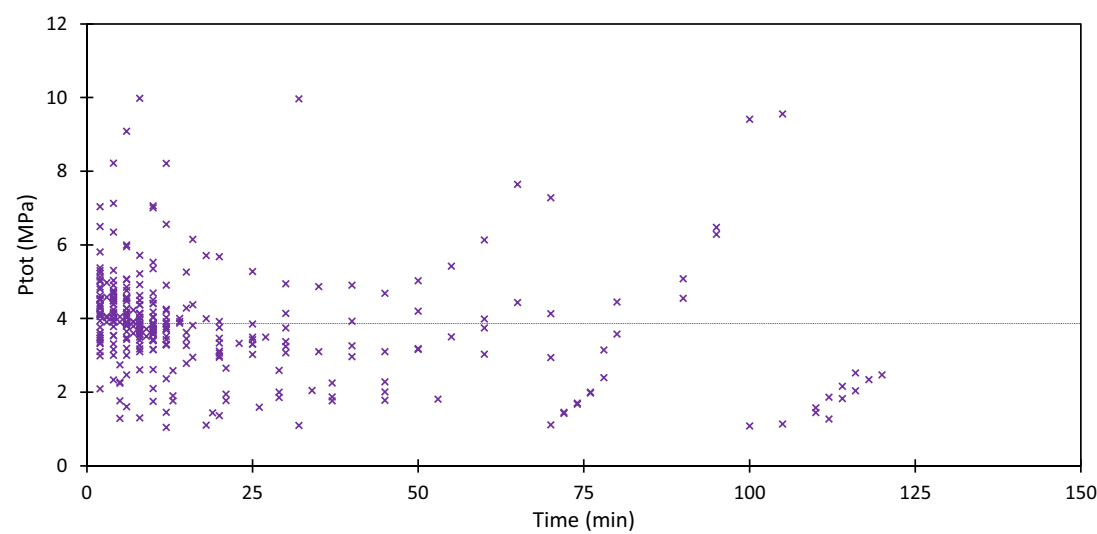




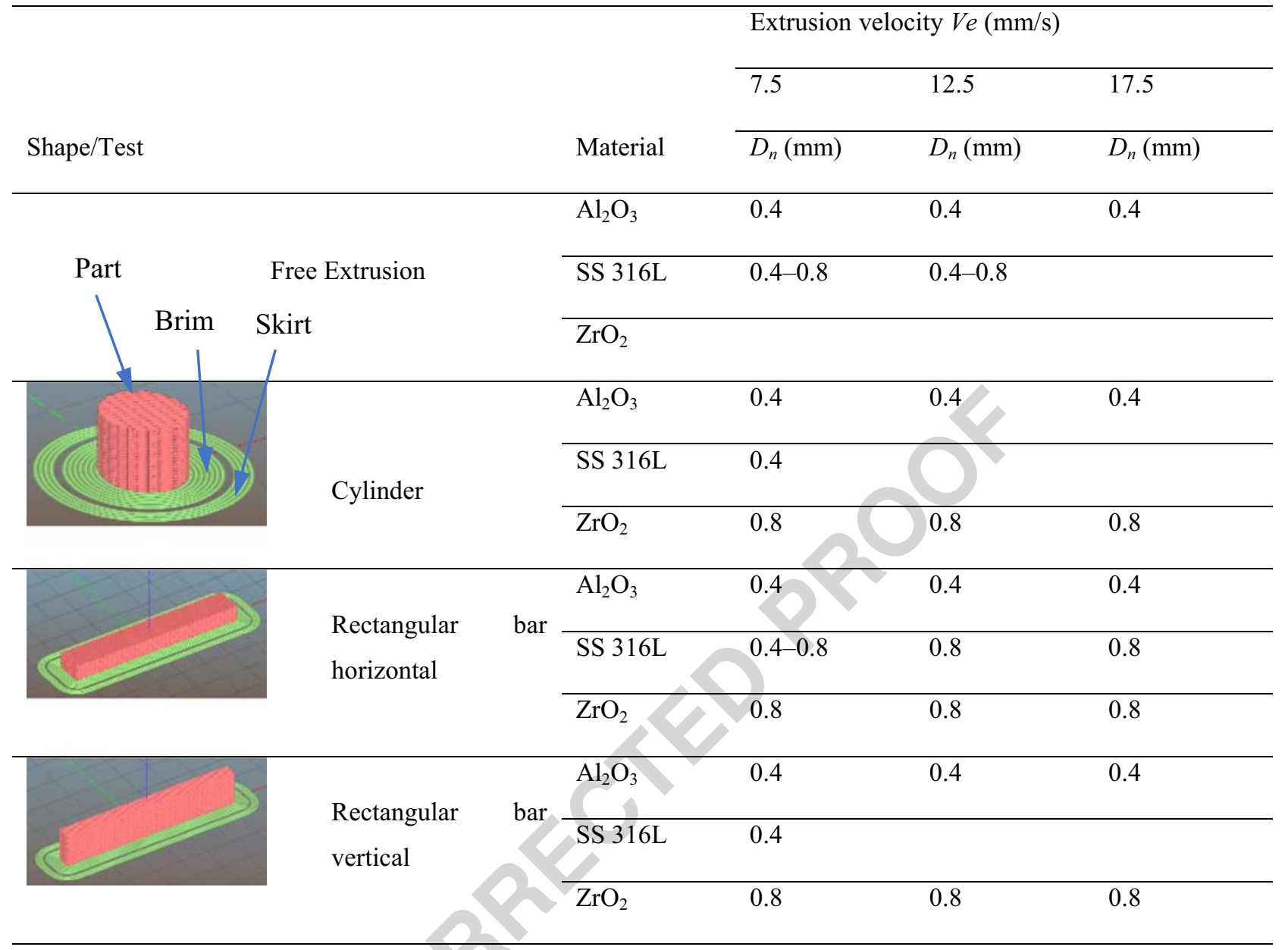

\section{Results and discussion of rheological data}

The rheological models given in Eqs. (9), (10) and (12) were applied to the capillary rheometer data (through linear regression). The corresponding material parameters $\left(\dot{\gamma}_{0}, K, n, l\right.$ and y) are provided in Table 4.

The values of consistency $l$ of the elongational viscosity are three orders of magnitude higher than the shear viscosity

Table 4 Power law parameters and correction shear rate for the three feedstocks consistency $K$ at a comparable strain rate. This means that the ratio of the elongational viscosity function to the shear viscosity function is high, corresponding to a Trouton ratio of $\eta_{E} / \eta_{\mathrm{s}}$. This Trouton ratio is approximately 100 in the case of $316 \mathrm{~L}$ feedstock and $200 \mathrm{~s}^{-1}$.

The consistency $K$ of the SS316L steel feedstock is significantly smaller than that of the other two materials at lower than 1200 Pa.s. Indeed, to verify the flow stability, samples of the extruded SS316L feedstock were collected at a shear rate of approximately $600 \mathrm{~s}^{-1}$. The surfaces of the rods are shown in Fig. 4a and b. For the left (longer) capillary, the quality observed at the outer surface of the rod is smooth, whereas

\begin{tabular}{llllrr}
\hline Feedstock & $K($ Pa.s $)$ & $n$ & $l$ (kPa.s) & $y$ & $\dot{\gamma}_{0}\left[\mathrm{~s}^{-1}\right]$ \\
\hline SS316L & 1187 & 0.678 & 1530 & 0.133 & 16 \\
Alumina & 5219 & 0.279 & 1086 & 0.210 & 1 \\
Zirconia & 3622 & 0.592 & 6568 & 0.050 & 7 \\
\hline
\end{tabular}



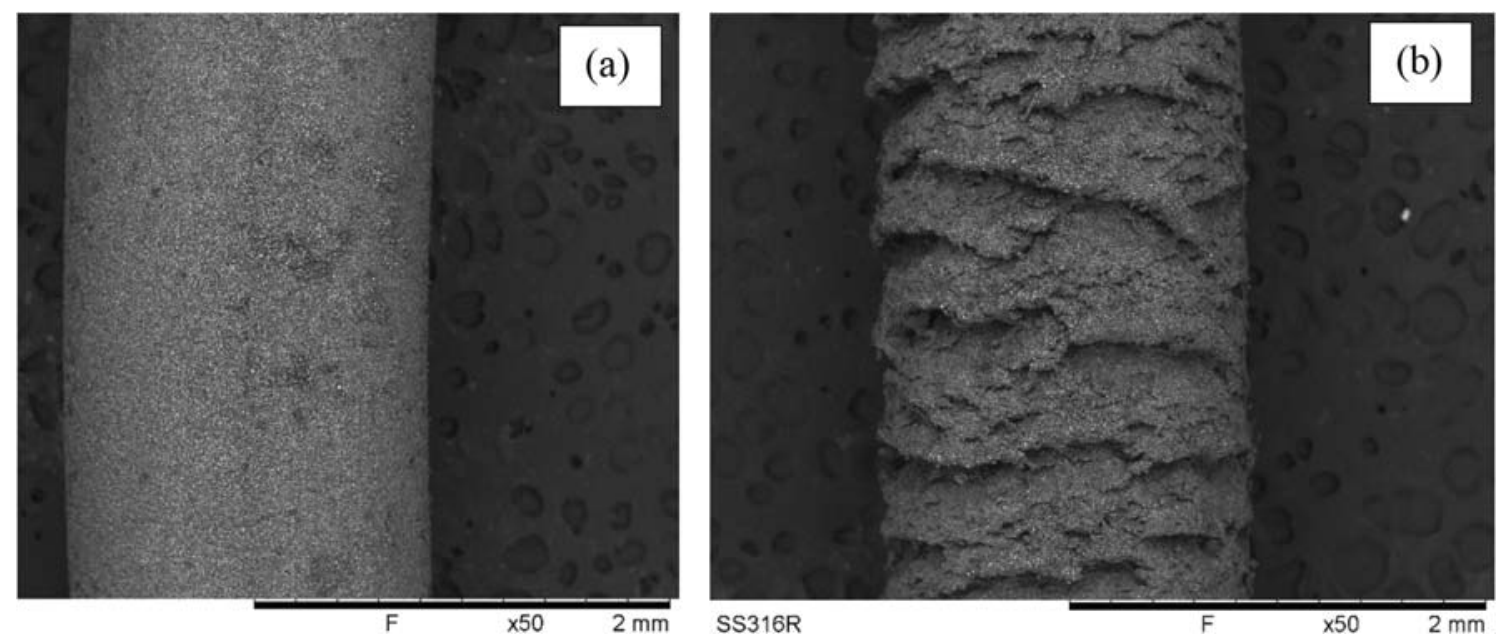

Fig. 4 Extruded roads of SS316L feedstock from $\mathbf{a}$ left and $\mathbf{b}$ right capillaries at shear rate of $600 \mathrm{~s}^{-1}$

the capillary extruded from the right (shorter) shows a sharkskin defect. This type of instability can be observed in a polymer extrusion when the capillary/nozzle length is extremely small $(L \approx 0)$ and is connected with a rapid detachment of the melt flow at the exit of the capillary [32]. This sharkskin problem has previously been observed for EAM processes [33].

The rheological models can be applied to the conditions of the planned extrusion and 3D printing tests (given in Table 3), and the corresponding expected feedstock viscosities at the nozzle can be estimated. A comparison of the shear and extensional viscosity characteristics of the three high loaded polymers corresponding to the extrusion and 3D printing test settings was conducted. In Fig. 5, a comparison between the shear and elongational viscosity is shown for all studied combinations. In the nozzle with $D_{\mathrm{n}}=0.8 \mathrm{~mm}$, a lower shear rate is clearly calculated. The shear rate tested with the alumina is larger owing to its lower $n$-value, which determines a stronger Rabinowitsch correction.

The elongational viscosity of the zirconia feedstock is significantly larger than that of the other two materials as compared with the shear viscosity, which is due to the larger elongational consistency $l$ for zirconia of greater than $6500 \mathrm{kPa} . \mathrm{s}$.

\subsection{Validation of pressure drop model}

The pressure drop model presented in Eq. (15) can be applied inversely, and if the viscosity values are known, the total pressure drops can be calculated. The rheological parameters given in Table 4 for the stainless steel feedstock were obtained from a Rosand capillary rheometer (rheometer A, $L_{1}=17 \mathrm{~mm}$, $L_{\mathrm{r}} \approx 0$ and $D_{1}=D_{\mathrm{r}}=1 \mathrm{~mm}$ ) and were used to predict the total pressure $P_{\text {tot }}$ required when using another rheometer with a different capillary configuration (rheometer $\mathrm{B}, L_{1}=32 \mathrm{~mm}$ and $D_{1}=D_{\mathrm{r}}=2 \mathrm{~mm}$ ). In Fig. 6 the predicted pressure is compared with the test with equipment $\mathrm{B}$, showing a good agreement. Rheometer B has a double length in the left capillary, but with nearly the same $L_{1} / D_{1}$ ratio, and hence the pressure requirement owing to the shear viscosity is similar. By contrast, the right bore of rheometer $\mathrm{B}$ has a double diameter and requires a significantly lower pressure $\Delta P_{\text {right }}$ owing to the elongational viscosity. In conclusion, the total pressure required by rheometer B is up to $40 \%$ smaller, primarily because
Fig. 5 Shear and elongational viscosity of three feedstocks versus shear rate corresponding to extrusion and $3 \mathrm{D}$ printing test settings
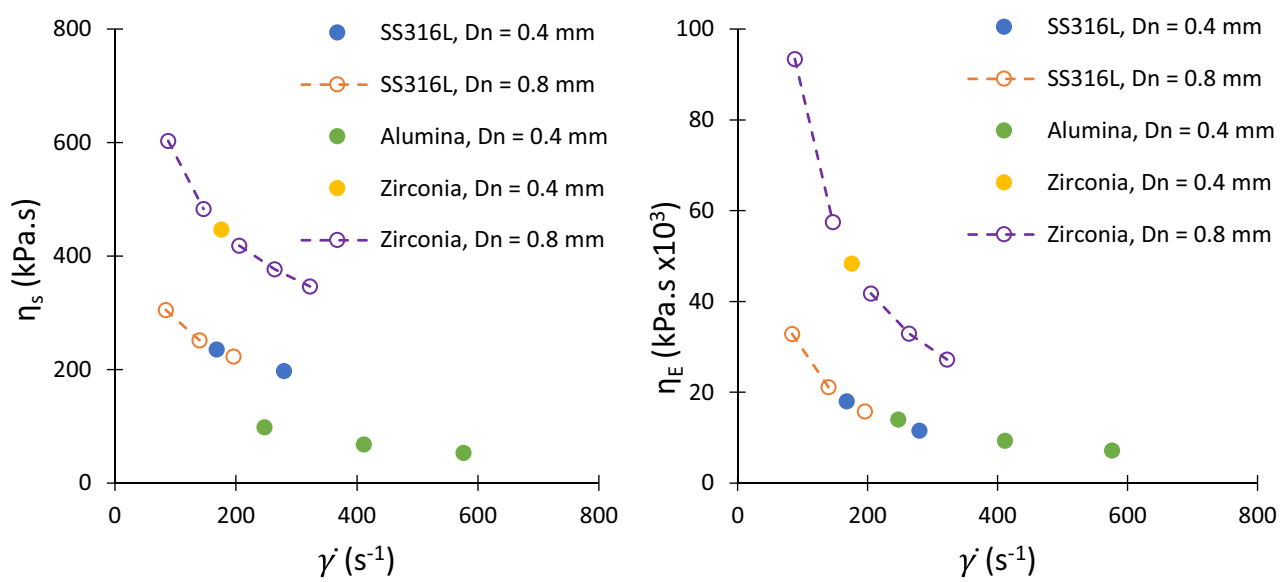


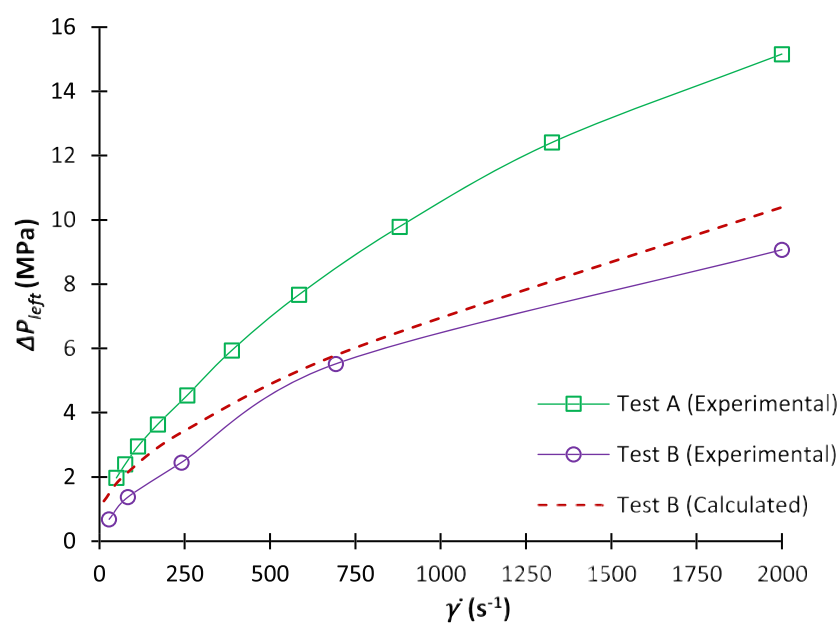

Fig. 6 Experimental and calculated $P_{\text {tot }}=\Delta P_{\text {left }}$ for different capillary configurations and strain rates of the elongational viscosity. This is an important confirmation of the important role of the elongational viscosity in the extrusion of powder-binder feedstocks.

\section{Results of extrusion and 3D printing tests}

The methodology used for predicting $P_{\text {tot }}=\Delta P_{\text {left }}$ with Eq. (15) at the left side capillary rheometer can also be adapted to predict the total extrusion pressure during extrusion and 3D printing tests on an EFeSTO machine. The extrusion unit is geometrically complex and can be reconducted into a series of cylindrical capillaries, the pressure drops of which can be estimated using Eq. (15).

Figure 7 shows a cross section of the flow channels of the extruder, with the respective pressure drop in the channels during the feedstock flows shown on the right side. From the main extruder chamber (A), the material flow is divided into two identical sections (B), which are further split into two long tubes (C) from each section. The material flow from these four channels flows into the nozzle region (D).

The cross-sectional area of the flow channels also accounts for the determination of the pressure drop through the extrusion unit and is subdivided into 11 zones with simple geometries. The elongational fraction of Eq. (15), $\Delta P_{\text {ent }}$, has been computed only for the entrance of sections 3, 5, 6 and 11 because these sections represent a restriction of the flow. In the shear fraction of Eq. (15), $\Delta P_{\text {cap }}$ has been measured at all sections after section 2, but is significant only at sections 6 and 11 because of the extremely high aspect ratio $L / D_{\mathrm{n}}$. The right side of Fig. 7 shows a representative plot of the pressure drop across the 11 sections measured at $V_{\mathrm{e}}=12.5 \mathrm{~mm} / \mathrm{s}$ and $D_{\mathrm{n}}=$ $0.4 \mathrm{~mm}$ for the alumina and steel feedstocks.

The total pressure drops under all experimental conditions were measured and compared with the experimental values. This comparison is summarized in Fig. 8, which shows that the measured pressure $\left(P_{\text {tot }}\right)$ and the calculated pressure components $\Delta P_{\text {cap }}$ and $\Delta P_{\text {ent }}$ increase linearly with an extrusion at velocity $V_{\mathrm{e}}$ because of the larger flow rate.
558

559

560

561

562

563

564

565

566

567

568

569

570

571

572

573

574

575

576

577

578

579

580

581

582

583

584

585
Fig. 7 Simplified cross-sectional view of extrusion system and flow channels used for feedstock

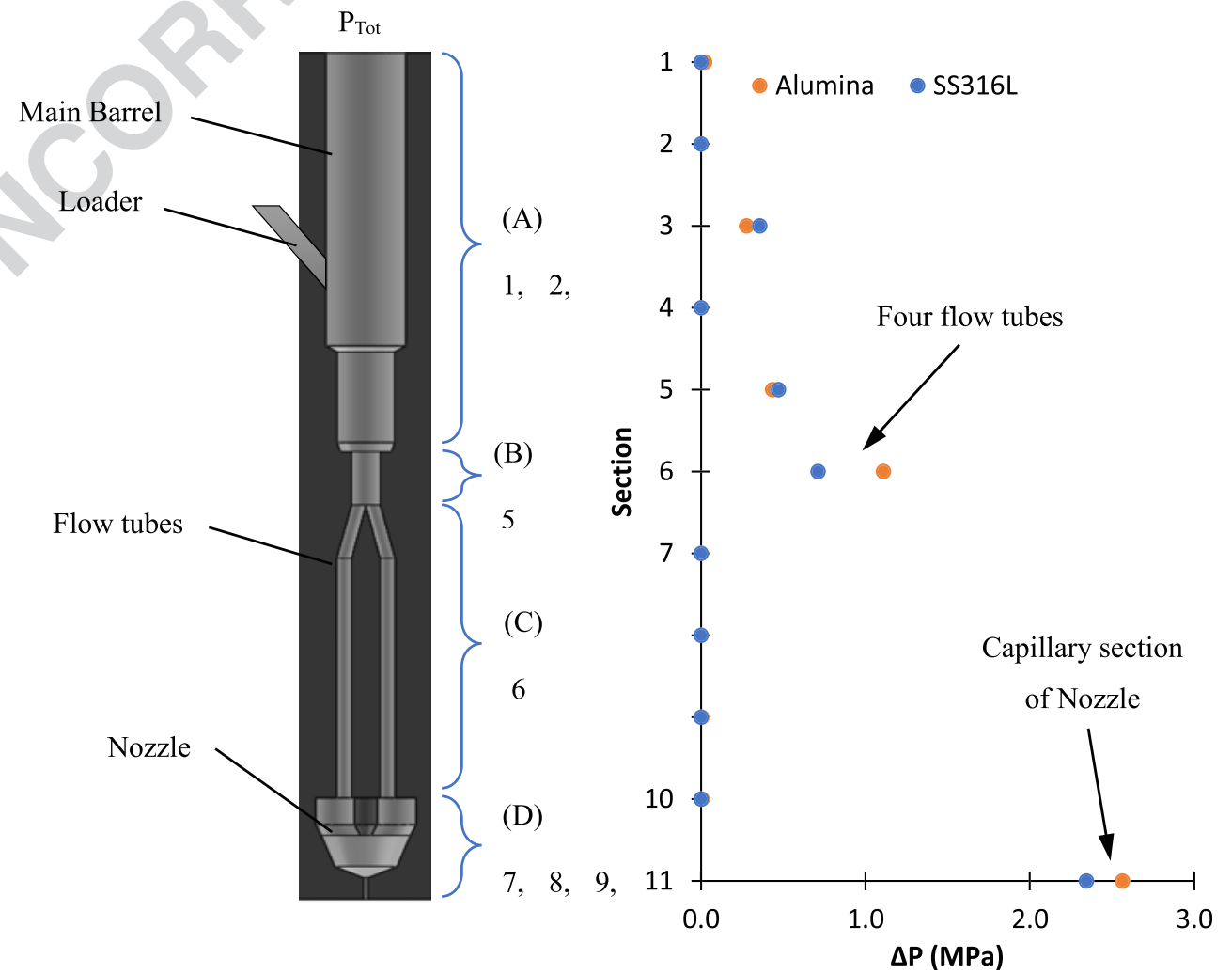


Int J Adv Manuf Technol

Fig. 8 Experimental and calculated extrusion pressures for different feedstocks and extrusion velocities; error bars are plotted equal to the pooled standard deviation of the $V_{\mathrm{e}}$ values of each graph
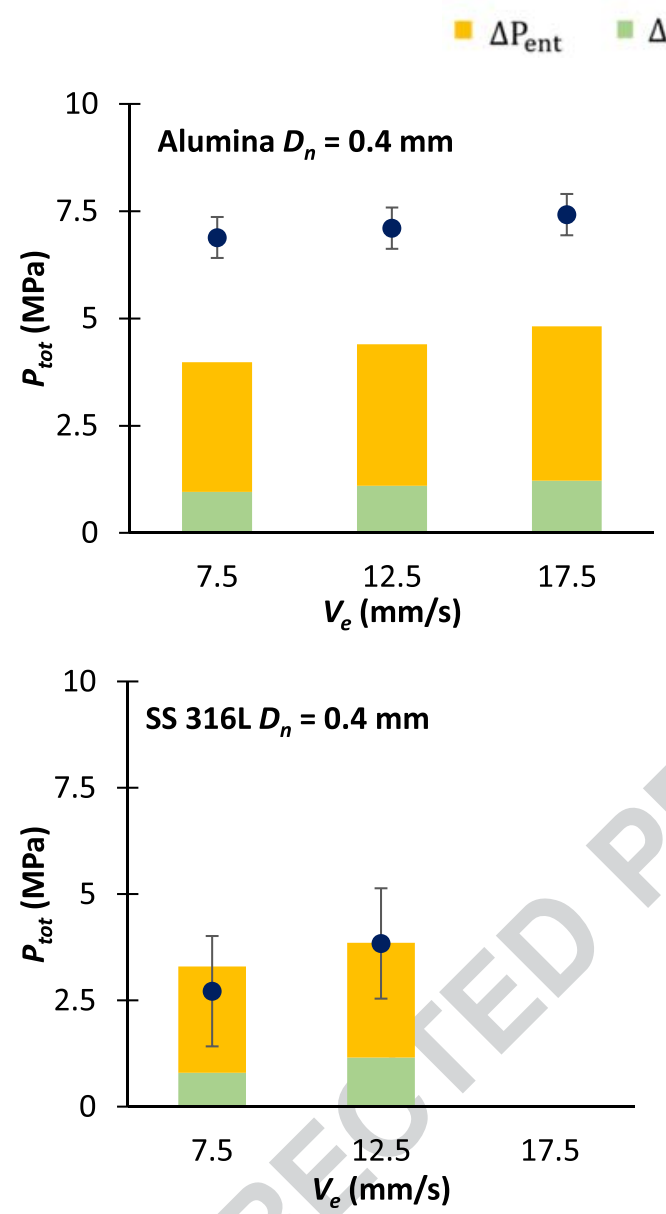

- $\mathrm{P}_{\text {tot }}$

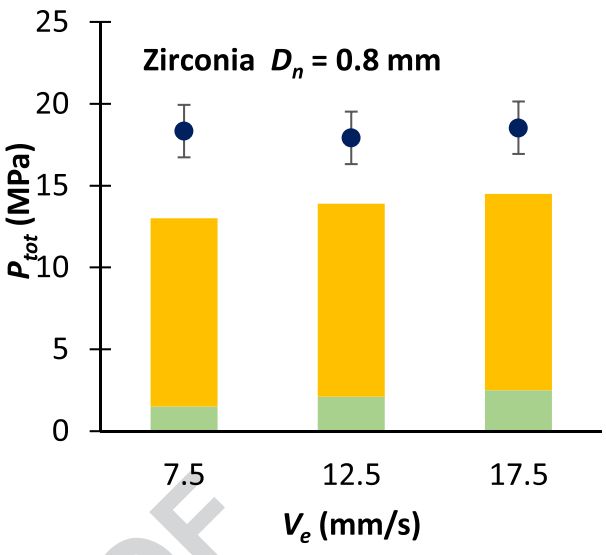

10

SS $316 \mathrm{~L} D_{n}=0.8 \mathrm{~mm}$

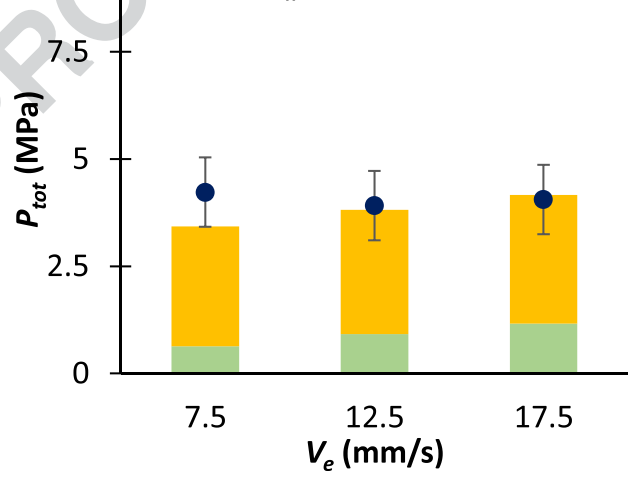

Zirconia requires more than double the pressure required by the alumina and SS316L. The alumina feedstock $P_{\text {tot }}$ requires between 6 and $8 \mathrm{MPa}$ of pressure, whereas the SS316L feedstock requires $P_{\text {tot }}$ at below $6 \mathrm{MPa}$. The predicted pressures properly capture the actual measure pressured for stainless steel, whereas they underestimate the actual pressure requirements of the two ceramic materials. This underestimation is probably connected with the different thermal values of the ceramic feedstock (having a larger thermal diffusivity, as shown in Table 2), and they likely cool faster than the steel as soon as they approach the exit of the nozzle, with a viscosity increase that cannot be captured by the model inside the extrusion unit. Although the model underestimates $P_{\text {tot }}$ for the ceramic feedstocks, it clearly gives an indication of the relative importance of the shear $\Delta P_{\text {cap }}$ and elongational $\Delta P_{\text {ent }}$ components of the pressure. Here, $\Delta P_{\text {cap }}$ is significantly larger
Fig. 9 Coefficient of variation $C O V_{\mathrm{P}}$ of the pressure signal for the different types of tests and materials

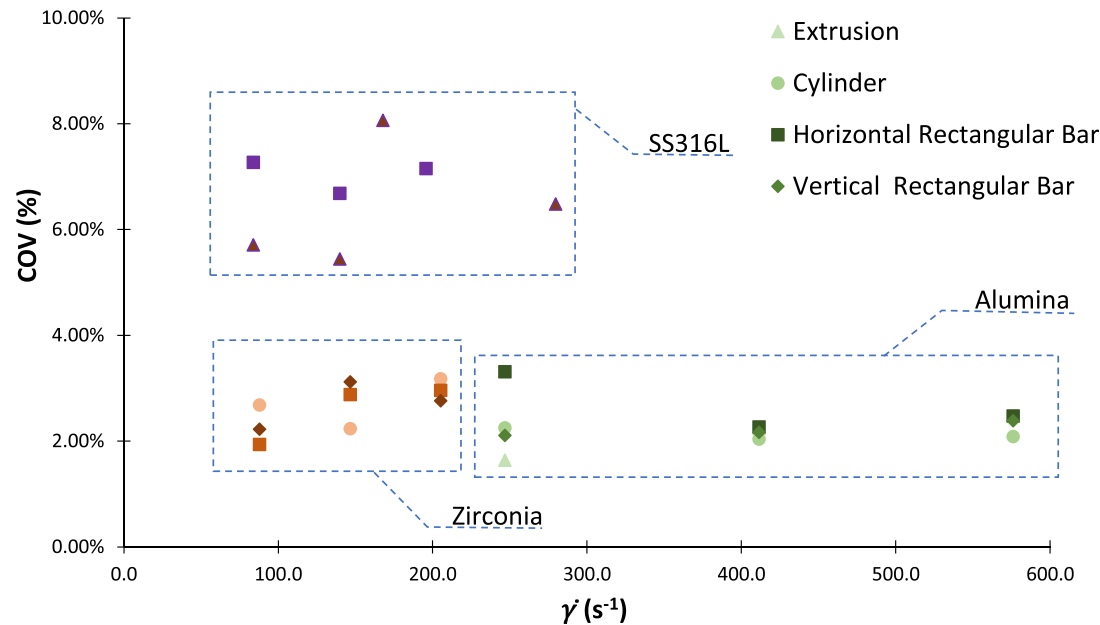


Fig. 10 Green and sintered alumina in rectangular bar-shaped parts printed in a vertical (top left) and horizontal (top right) configuration and green and sintered steel (bottom right) and zirconia (bottom left) parts

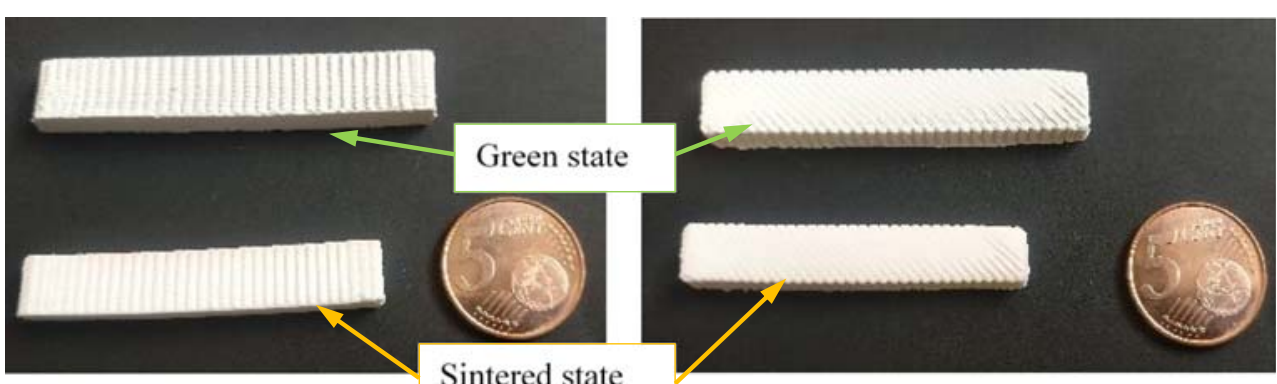

Sintered state
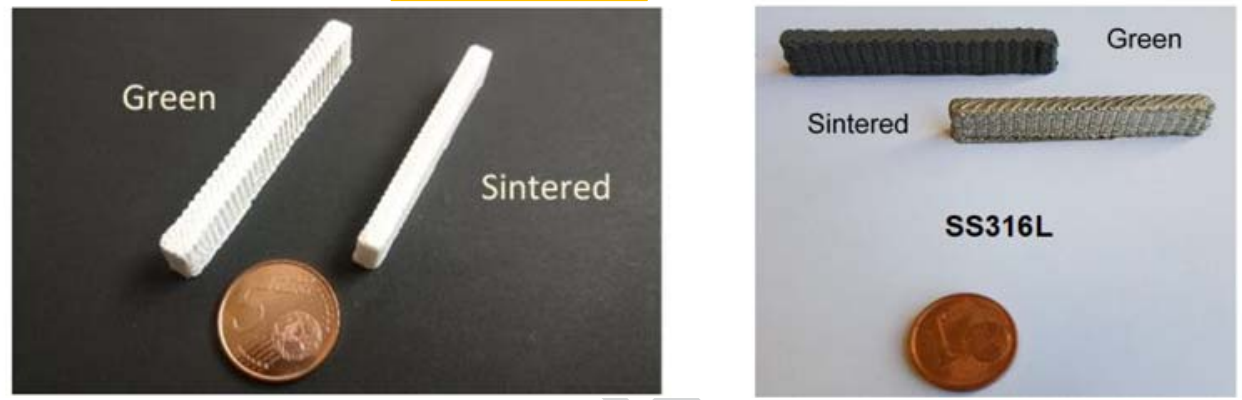

t5.1 Table 5 Sintered properties of alumina, zirconia and S316L at extrusion speed of $12.5 \mathrm{~mm} / \mathrm{s}$

\begin{tabular}{lllll}
\cline { 2 - 4 } t5.2 & Material & Alumina & Zirconia & SS316L \\
\cline { 2 - 4 } t5.3 & Density $\left(\mathrm{g} / \mathrm{cm}^{3}\right)$ & 3.60 & 5.65 & 7.11 \\
t5.4 & Elastic modulus $(\mathrm{GPa})$ & 81.5 & 27.33 & 77.26 \\
\cline { 2 - 4 }
\end{tabular}

By contrast, the error $C O V_{\mathrm{P}}$ in the pressure signal measured using the four different types of printing (free extrusion, cylinder, horizontal prismatic bar and vertical prismatic bar) did not show any clear differences, with a random ranking among the different shapes. This is effectively shown through Fig. 9. The reason for the lower stability of the steel is probably connected to its lower consistency $K$, as a confirmation of previous findings [6]. Interestingly, there seems to be no correlation between the shear rate and the stability $C O V_{\mathrm{P}}$ of the pressure signal.

After 3D printing, some of the samples underwent debinding and were sintered to better understand the variations in their surface quality characteristics. Because this study is focused on the extrusion pressure, the parts were printed without outer contour roads to enhance the variations owing to the start and stops and directional changes. All samples therefore show an extremely rough surface finish in a green state, which mildly improves after sintering. Representative 3D printed parts in their green and sintered states are shown in Fig. 10, and their sintered properties are reported in Table 5.

Owing to the sintering, shrinkage reduces the waviness on the surface, although the structure of the surface texture remains unchanged. To further recognize the role of the printing parameters on the surface quality, surface characteristics of 3D printed components are also analysed using SEM. The observations indicate that the surface quality of the components is not correlated with $C O V_{\mathrm{P}}$ and depends only on the material to be printed and on the infill and layering parameters, as is wellknown for all EAM processes. As an example, Fig. 11 a to $\mathrm{c}$ compare the surface characteristics of green SS316L samples printed with different layer heights $h$. Because the surface quality of the parts is not influenced by the variations in pressure, a quantitative report of the surface quality data is omitted 

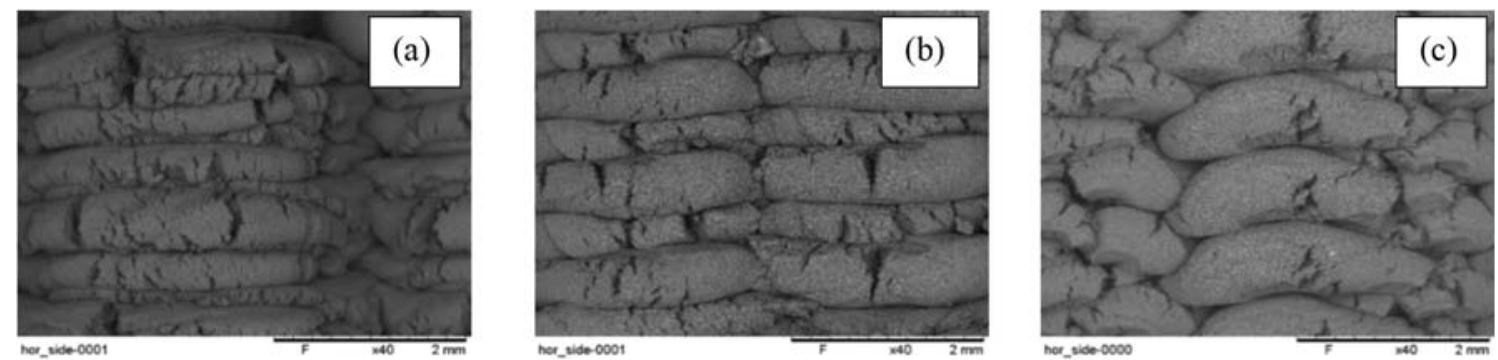

Fig. 11 SEM images for side walls of SS316L samples, printed with a 0.8 - $\mathrm{mm}$ nozzle and different printed layer heights $h$ of a 0.3 , b 0.4 and $\mathbf{c} 0.5 \mathrm{~mm}$

herein for brevity. Zhou et al. developed some numerical optimisation approaches to increase the tensile strength and control the volumetric shrinkage values through different cost functions dedicated to the polymer FFD process and optimized the processing parameters [36]. This promising method will be adapted in the future. An estimation of the shrinkage is a real challenge in additive manufacturing, and Fotovvati et al. proposed an analytical expression to quantify the size dependency of the dimensional percentage errors with a polynomial function in the DMLS manufactured features [37]. This methodology will be adjusted during the FFD process in the future.

\section{Conclusions}

This work is focused on the measurement and prediction of the instantaneous pressure occurring during the extrusion and EAM operations of highly viscous powder-binder feedstocks. A pressure prediction model was developed when considering both the shear and elongational viscosity contributions. The material parameters were calculated from capillary rheometry data, which were also used to validate the model by verifying its agreement with the experimental viscosity measurements.

An extensive extrusion plan and $3 \mathrm{D}$ printing tests were applied to three different materials (steel, alumina and zirconia) over a range of different nozzle diameters, extrusion velocities and 3D printed shapes. The results indicate that the pressure requirements owing to the elongational viscosity are dominant with respect to the contributions of the shear viscosity.

The results also indicate that, among the investigated parameters, the stability of the pressure signals depends on the material feedstock and not on the shear rate or shape of the 3D printed parts.

\section{References}

1. Royer A, Barrière T, Gelin JC (2016) Development and characterization of a metal injection molding bio sourced inconel 718 feedstock based on polyhydroxyalkanoates. Metals (Basel) 6. https:// doi.org/10.3390/met6040089
2. Rane K, Strano M (2019) A comprehensive review of extrusionbased additive manufacturing processes for rapid production of metallic and ceramic parts. Adv Manuf 7:155-173. https://doi.org/ 10.1007/s40436-019-00253-6

3. Chen Z, Li Z, Li J, Liu C, Lao C, Fu Y, Liu C, Li Y, Wang P, He Y (2019) 3D printing of ceramics: a review. J Eur Ceram Soc 39:661687. https://doi.org/10.1016/j.jeurceramsoc.2018.11.013

4. Nadernezhad A, Unal S, Khani NKB (2019) Material extrusionbased additive manufacturing of structurally controlled poly(lactic acid)/carbon nanotube nanocomposites. Int J Adv Manuf Technol 102:2119-2132

5. Annoni M, Giberti H, Strano M (2016) Feasibility study of an extrusion-based direct metal additive manufacturing technique. Procedia Manuf 5:916-927. https://doi.org/10.1016/j.promfg. 2016.08.079

6. Strano M, Rane K, Briatico Vangosa F, Di Landro L (2019) Extrusion of metal powder-polymer mixtures: melt rheology and process stability. J Mater Process Technol 273:116250. https://doi. org/10.1016/j.jmatprotec.2019.116250

7. Hidalgo J, Jiménez-Morales A, Barriere T, Gelin JC, Torralba JM (2015) Capillary rheology studies of INVAR 36 feedstocks for powder injection moulding. Powder Technol 273:1-7. https://doi. org/10.1016/j.powtec.2014.12.027

8. Dimitri C, Mohamed S, Thierry B, Jean-Claude G (2017) Influence of particle-size distribution and temperature on the rheological properties of highly concentrated Inconel feedstock alloy 718 . Powder Technol 322:273-289. https://doi.org/10.1016/j.powtec. 2017.08.049

9. Rane K, Di Landro L, Strano M (2019) Processability of SS316L powder - binder mixtures for vertical extrusion and deposition on table tests. Powder Technol 345:553-562. https://doi.org/10.1016/j. powtec.2019.01.010

10. Turner BN, Strong RA, Gold S (2014) A review of melt extrusion additive manufacturing processes: I. Process design and modeling. Rapid Prototyp J 20:192-204. https://doi.org/10.1108/RPJ-012013-0012

11. Mackay ME (2018) The importance of rheological behavior in the additive manufacturing technique material extrusion. J Rheol (N Y N Y) 62:1549-1561. https://doi.org/10.1122/1.5037687

12. Singh P, Shaikh Q, Balla VK, Atre SV, Kate KH (2019) Estimating powder-polymer material properties used in design for metal fused filament fabrication (DfMF 3). JOM. https://doi.org/10.1007/ s11837-019-03920-y

13. Faes M, Vleugels J, Vogeler F, Ferraris E (2016) Extrusion-based additive manufacturing of $\mathrm{ZrO} 2$ using photoinitiated polymerization. CIRP J Manuf Sci Technol 14:28-34. https://doi.org/10.1016/ j.cirpj.2016.05.002

14. Kukla C, Gonzalez-Gutierrez J, Duretek I, Schuschnigg S, Holzer C (2017) Effect of particle size on the properties of highly-filled polymers for fused filament fabrication. AIP Conf Proc 1914. https:// doi.org/10.1063/1.5016795

15. Khaliq MH, Gomes R, Fernandes C, Nóbrega J, Carneiro OS, Ferrás LL (2017) On the use of high viscosity polymers in the fused 
filament fabrication process. Rapid Prototyp J 23:727-735. https:// doi.org/10.1108/RPJ-02-2016-0027

16. Coogan TJ, Kazmer DO (2019) In-line rheological monitoring of fused deposition modeling. J Rheol (N Y N Y) 63:141-155. https:// doi.org/10.1122/1.5054648

17. Thavanayagam G, Pickering KL, Swan JE, Cao P (2015) Analysis of rheological behaviour of titanium feedstocks formulated with a water-soluble binder system for powder injection moulding. Powder Technol 269:227-232. https://doi.org/10.1016/j.powtec. 2014.09.020

18. Khakbiz M, Simchi A, Bagheri R (2005) Analysis of the rheological behavior and stability of 316L stainless steel-TiC powder injection molding feedstock. Mater Sci Eng A 407:105-113. https:// doi.org/10.1016/j.msea.2005.06.057

19. Park SJ, Kim D, Lin D, Park SJ, Ahn S (2017) Rheological characterization of powder mixture including a space holder and its application to metal injection molding. Metals (Basel) 7. https:// doi.org/10.3390/met7040120

20. Huang B, Liang S, Qu X (2003) The rheology of metal injection molding. J Mater Process Technol 137:132-137. https://doi.org/10. 1016/S0924-0136(02)01100-7

21. Samanta SK, Chattopadhyay H, Godkhindi MM (2011) Thermophysical characterization of binder and feedstock for single and multiphase flow of PIM 316L feedstock. J Mater Process Technol 211:2114-2122. https://doi.org/10.1016/j.jmatprotec.2011.07.008

22. Huang JC, Leong KS (2002) Shear viscosity, extensional viscosity, and die swell of polypropylene in capillary flow with pressure dependency. J Appl Polym Sci 84:1269-1276. https://doi.org/10. 1002/app.10466

23. Aho J, Syrjälä S (2011) Shear viscosity measurements of polymer melts using injection molding machine with adjustable slit die. Polym Test 30:595-601. https://doi.org/10.1016/j.polymertesting. 2011.04.014

24. Ohtani H, Ellwood K, Pereira G, Chinen T, Selvasekar S (2017) Extensional rheology: new dimension of characterizing automotive fluids. SAE Tech Pap. https://doi.org/10.4271/2017-01-0364

25. Cogswell FN (1972) Measuring the extensional rheology of polymer melts. Trans Soc Rheol 16:383-403. https://doi.org/10.1122/1. 549257

26. Zatloukal M, Musil J (2009) Analysis of entrance pressure drop techniques for extensional viscosity determination. Polym Test 28:843-853. https://doi.org/10.1016/j.polymertesting.2009.07.007
27. Hong SY, Broomer M (2000) Economical and ecological cryogenic machining of AISI 304 austenitic stainless steel. Clean Prod Process 2:0157-0166. https://doi.org/10.1007/s100980000073

28. Arabo EYM (2011) Shear and extensional viscosities of hard wheat flour dough using a capillary rheometer. J Food Eng 103:294-298. https://doi.org/10.1016/j.jfoodeng.2010.10.027

29. Parenti P, Cataldo S, Grigis A, Covelli M, Annoni M (2019) Implementation of hybrid additive manufacturing based on extrusion of feedstock and milling. Procedia Manuf 34:738-746. https:// doi.org/10.1016/j.promfg.2019.06.230

30. Heaney DF (2012) Handbook of Metal Injection Molding, 1st edn. Woodhead Publishing

31. Liu ZY, Loh NH, Tor SB, Khor KA (2003) Characterization of powder injection molding feedstock. Mater Charact 49:313-320. https://doi.org/10.1016/S1044-5803(02)00282-6

32. Vergnes B (2015) Extrusion defects and flow instabilities of molten polymers. Int Polym Process 30:3-28. https://doi.org/10.3139/217. 3011

33. Kishore V, Ajinjeru C, Liu P, Lindahl J, Hassen A, Kunc V et al (2017) Predicting sharkskin instability in extrusion additive manufacturing of reinforced thermoplastics. Solid Free Fabr Symp:1696-1704

34. Rane K, Petrò S, Strano M (2020) Evolution of porosity and geometrical quality through the ceramic extrusion additive manufacturing process stages. Addit Manuf 32:101038. https://doi.org/10. 1016/j.addma.2020.101038

35. Strano M, Rane K, Herve G, Tosi A (2019) Determination of process induced dimensional variations of ceramic parts, $3 \mathrm{~d}$ printed by extrusion of a powder-binder feedstock. Procedia Manuf 34:560 565. https://doi.org/10.1016/j.promfg.2019.06.220

36. Zhou X, Hsieh SJ, Wang JC (2019) Accelerating extrusion-based additive manufacturing optimization processes with surrogatebased multi-fidelity models. Int J Adv Manuf Technol 103:40714083

37. Fotovvati BAE (2019) Size effects on geometrical accuracy for additive manufacturingof Ti-6Al-4V ELI parts. Int J Adv Manuf Technol 104:2951-2959

Publisher's note Springer Nature remains neutral with regard to jurisdictional claims in published maps and institutional affiliations. 


\section{AUTHOR QUERIES}

\section{AUTHOR PLEASE ANSWER ALL QUERIES.}

Q1. Please check if the edits to the sentence starting "ning continuous extrusion..." retained the intended meaning. Otherwise, kindly amend.

Q2. Figures 4,11 contains poor quality and small text inside the artwork. Please do not re-use the file that we have rejected or attempt to increase its resolution and re-save. It is originally poor, therefore, increasing the resolution will not solve the quality problem. We suggest that you provide us the original format. We prefer replacement figures containing vector/editable objects rather than embedded images. Preferred file formats are eps, ai, tiff and pd 